\title{
Prioritization of osteoporosis-associated GWAS SNPs using epigenomics and transcriptomics
}

\author{
Xiao Zhang ${ }^{1}$, Hong-Wen Deng ${ }^{2}$, Hui Shen ${ }^{1}$, and Melanie Ehrlich ${ }^{3 *}$
}

${ }^{1}$ Department of Biostatistics and Data Science, School of Public Health and Tropical Medicine, Tulane University, New Orleans, LA, USA; ${ }^{2}$ Tulane Center for Biomedical Informatics and Genomics, Division of Biomedical Informatics and Genomics, Deming Department of Medicine, School of Medicine, Tulane University, New Orleans, LA, USA; ${ }^{3}$ Tulane Cancer Center and Hayward Genetics Center, Tulane University, New Orleans, LA, USA

\section{*Corresponding author}

Melanie Ehrlich

Professor,

Tulane Cancer Center and Hayward Genetics Center

Tulane University Health Sciences Center

New Orleans, LA 70112

Email: ehrlich@tulane.edu

Phone: 504-988-2449

Key words: osteoporosis, osteoblasts, epigenetics, molecular pathways - remodeling, Wnt/ $\beta$-catenin 


\section{Abstract}

Genetic risk factors for osteoporosis, a prevalent disease associated with aging, have been examined in many genome-wide association studies (GWAS). A major challenge is to prioritize transcription-regulatory GWAS-derived variants that are likely to be functional. Given the critical role of epigenetics in gene regulation, we have used an unusual epigenetics- and transcription-based approach to identify credible regulatory SNPs relevant to osteoporosis from 38 reported BMD GWAS. Using Roadmap databases, we prioritized SNPs based upon their overlap with strong enhancer or promoter chromatin preferentially in osteoblasts relative to 11 heterologous cell culture types. The selected SNPs also had to overlap open chromatin (DNaseI-hypersensitive sites) and DNA sequences predicted to bind to osteoblast-relevant transcription factors in an allele-specific manner. From >50,000 GWAS-derived SNPs, we identified 16 novel and credible regulatory SNPs (Tier-1 SNPs) for osteoporosis risk. Their associated genes, BICC1, LGR4, DAAM2, NPR3, or HMGA2, are involved in osteoblastogenesis or bone homeostasis and regulate cell signaling or enhancer function. Four of them are preferentially expressed in osteoblasts. BICC1, LGR4, and DAAM2 play important roles in canonical Wnt signaling, a pathway critical to bone formation and repair. The transcription factors that are predicted to bind to the Tier-1 SNP-containing DNA sequences also have bone-related functions. For the seven Tier-1 SNPs near the 5' end of $B I C C 1$, examination of eQTL overlap and the distribution of BMD-increasing alleles suggests that at least one SNP in each of two clusters contributes to inherited osteoporosis risk. Our study not only illustrates a method that can be used to identify novel BMD-related causal regulatory SNPs for future study, but also reveals evidence that some of the Tier-1 SNPs exert their effects on BMD risk indirectly through little-studied noncoding RNA genes, which in turn may control the nearby bone-related proteinencoding gene. 


\section{Introduction}

Osteoporosis, which affects about 200 million people worldwide, involves a loss of bone mass, strength, and normal microarchitecture. ${ }^{(1)}$ Both osteoporosis and bone fracture increase greatly with age. ${ }^{(2)}$ Bone mineral density (BMD) of the hip, spine, limb, or total body is the quantitative trait used most frequently to detect or predict osteoporosis. ${ }^{(1,3)}$ In the clinic, BMD is usually determined by dual-energy x-ray absorptiometry (DXA). In genome-wide association studies (GWAS), the simpler-to-perform ultrasound-measured heel bone density (estimated BMD, eBMD) is often used. Like DXA-determined BMD, eBMD has a high heritability $(50-80 \%)$ and can predict bone fracture after correcting for the effects of height, weight, age, and sex. ${ }^{(4)}$ Even low-frequency variants (minor allele frequency of 1 - 5\%) or rare variants may contribute to decreased BMD and increased bone fracture. ${ }^{(5)}$

From DXA or eBMD GWAS (collectively referred to here as BMD GWAS), hundreds of genes have been identified as having nearby or overlapping genetic variants significantly associated with osteoporosis risk. ${ }^{(1,4,6)}$ Most BMD or other trait-associated variants are located in non-coding regions of the genome. ${ }^{(1,7)}$ Identifying causal osteoporosis-risk genes and their associated variants, usually SNPs, is challenging for variants that are not missense coding variants. The GWAS-derived SNP exhibiting the smallest p-value for association with the studied trait at a given locus (index SNP) is often linked to very many proxy SNPs solely because of linkage disequilibrium (LD) rather than due to biological relevance. Recently, whole-genome profiles of epigenetics features, such as overlap with open chromatin (e.g., DNaseI hypersensitive sites, DHS), have been used to help discriminate likely causal BMD regulatory variants from bystander variants in high LD with them. ${ }^{(5,6,8-10)}$ Moreover, GWAS SNPs are generally also enriched in enhancer or promoter chromatin. ${ }^{(7,10)}$ The importance of considering such epigenetic features at BMD GWAS-derived SNPs is also evidenced by the finding that specific epigenetic changes play a key role in bone formation and remodeling. ${ }^{(11,12)}$ 
Using epigenetics to help prioritize BMD GWAS variants requires choosing the cell types for study. Bone has one of the most complex developmental and repair pathways due in part to its rigidity, strength, highly dynamic nature, and multiple structural and physiological roles. ${ }^{(13)}$ Osteoblasts (ostb) play a central role in both intramembranous ossification and in the more widespread and complicated endochondral ossification pathway for bone formation based upon chondrocytes (chond). ${ }^{(11)}$ Surprisingly, there is evidence for hypertrophic chond transdifferentiating to ostb, which is consistent with the considerable overlap of transcription profiles of these two morphologically dissimilar bone cell types. ${ }^{(14,}$ ${ }^{15)}$ Ostb and chond are derived from a heterogeneous mixture of bone-derived stem or stem-like cells commonly referred to as mesenchymal stem cells (MSC). ${ }^{(13)}$ Bone-destroying osteoclasts, which arise from the monocyte blood lineage, contribute to osteoporosis when bone resorption is excessive. Remodeling of cortical bone occurs through complex multicellular units containing ostb and osteoclasts, which modulate each other's function and are often responsive to some of the same growth factors and cytokines, although sometimes with different responses. ${ }^{(2,16)}$ Osteocytes (terminally differentiated ostb), which constitute most of the cells in cortical bone, coordinate the differentiation and activity of ostb and osteoclasts in response to mechanical load or injury. ${ }^{(17)}$

Because of the centrality of ostb to normal bone homeostasis and the availability of epigenomic and transcriptomic profiles for comparing human cell cultures of ostb, chond, MSC, and heterologous primary cell cultures, we focused on examining the epigenetics of BMD GWAS-derived SNPs in ostb and secondarily, in chond and MSC. In a recent study, we used epigenetics to prioritize best-candidate causal variants for BMD GWAS SNPs and obesity GWAS SNPs at a single gene, TBX15, which encodes a limb development-associated transcription factor $(\mathrm{TF}){ }^{(18)}$ In the present study, we take the unusual approach of looking genome-wide not only for SNPs overlaying enhancer or promoter chromatin in ostb but also for such SNPs that displayed these types of regulatory chromatin preferentially in ostb relative to many types of cell cultures not directly related to bone. Using epigenomics, transcriptomics, gene ontology (GO) 
analysis, and TF binding site (TFBS) prediction in a analysis of whole-genome data from 38 BMD GWAS, we identified 16 high-priority candidates for causal regulatory SNPs associated with five bonerelevant genes (BICC1, NPR3, LGR4, HMGA2, and DAAM2). None of these SNPs was previously described as associated with osteoporosis risk.

\section{Materials and Methods}

\section{BMD GWAS-derived SNPs}

Index SNPs associated with BMD $\left(p<5 \times 10^{-8}\right)$ were retrieved from 38 studies in the NHGRI-EBI GWAS Catalog $^{(19)}$ (downloaded October 2019; Supplemental Table S1). We expanded the index SNPs to a set of proxy SNPs $\left(r^{2} \geq 0.8\right.$, EUR from the 1000 Genome Project Phase $\left.3^{(20)}\right)$ by using PLINK v1.9(21) (https://www.cog-genomics.org/plink/1.9/) and/or LDlink v3.9 ${ }^{(22)}$ (https://ldlink.nci.nih.gov/). For the five best candidate genes, we also extracted imputed SNPs $\left(p<5 \times 10^{-8}\right)$ for total-body DXA BMD ${ }^{(3)}$ and $(p<$ $\left.6.6 \times 10^{-9}\right)$ for $\mathrm{eBMD}^{(6)}$ to look for additional Tier-1 SNPs.

\section{Transcriptomics}

For determining preferential expression of genes in ostb we obtained RPKM (reads per kilobase million) for ostb and 11 heterologous cell cultures (Supplemental Table S2), not including MSC or chond, from the ENCODE RNA-seq database (Supplemental Methods; http://genome.ucsc.edu). Preferential expression was defined as the ratio of ostb RPKM/median non-ostb RPKM > 5 and with a minimum of RPKM > 1 for ostb. The differentiation status of the commercially obtained ostb that had been used to generate the ENCODE RNA-seq data as well as for the epigenomic data (see below) is unclear. However, expression and chromatin segmentation profiles for SP7, RUNX2, SPP1, IBSP, TNFRSF11B, BGLAP, and $A L P L$ indicate selective transcription of middle- and late ostb differentiation marker genes by ostb 
samples and chond-specific expression of SOX5 in chond samples (Supplemental Figs. S1 and S2). For some genes, we also examined tissue expression profiles (median transcripts per million, TPM, and tissue expression QTL, eQTL) from the GTEx database ${ }^{(23)}$ (https://www.gtexportal.org/) and mouse expression microarray profiles from BioGPS ${ }^{(24)}$ (http://biogps.org/).

\section{Epigenomics}

Chromatin state segmentation data for the 15 examined cell culture types (Supplemental Methods and Table S3) were obtained from the 18-state Roadmap model with strong promoter chromatin as state 1 and strong enhancer chromatin as states 3,8 or 9 (Roadmap $^{(7)}$; http://genome.ucsc.edu). In figures for clarity in depicting these data, we slightly modified the color-coding of the chromatin state segmentation as indicated. DHS were defined as narrowPeaks from DNase-seq (Roadmap ${ }^{(7)}$; $\underline{\text { htp://genome.ucsc.edu) }}$.

\section{Predictions of allele-specific transcription factor binding sites and analyses of gene functional terms}

Predictions of allele-specific TFBS were made using a 21-base sequence centered around the SNP of interest for comparison to the TRANSFAC v2019.3 database (Supplemental Methods). We checked that each TF for a matching TFBS had appreciable expression in ostb (RPKM $\geq 0.8$ ). In addition, we used manual curation to retain only those TRANSFAC TFBS predictions for which all of the conserved positions $(5-10)$ in the PWM (position weight matrix) had exact matches to those in the SNP-containing sequence and no more than one base in a partly conserved position had only a partial match (at least $20 \%$ as good as the best PWM match). For determining that a TFBS overlapping a SNP is likely to be allelespecific in its TF binding, we required that the disfavored allele had a position probability that was at least 5-fold lower than that of the favored allele in the PWM matrix. DAVID v6.7 ${ }^{(25)}$ (https://davidd.ncifcrf.gov/) was used for functional classification of reference genes and GREAT v4.0.4 ${ }^{(26)}$ (http://great.stanford.edu/) for prioritization of genes linked to our epigenetically-selected GWAS SNP subset. 


\section{Results}

\section{Prioritization of 16 SNPs as candidate causal regulatory SNPs (Tier-1 SNPs) from 38 BMD GWAS}

To identify high-priority candidates for causative regulatory variants from BMD GWAS, we obtained index SNPs from 38 eBMD or DXA BMD studies (Fig. 1; Supplemental Table S1) and expanded them (LD threshold of $r^{2} \geq 0.8$ ) using the European ancestry (EUR) population because it was the predominant one studied. As expected, the GO term with the most enrichment among the 2,157 reference genes associated with the BMD GWAS index SNPs was skeletal system development (Supplemental Table S4). In accord with other analyses, ${ }^{(1,7)}$ only a small percentage $(2 \%, 1,139$ SNPs) of the 57,235 BMD-related index/proxy SNPs mapped to coding regions, and they were associated with $29 \%$ of the reference genes (Supplemental Table S3).

In order to prioritize GWAS SNPs for the best causative regulatory candidates, we first looked for SNPs that preferentially overlap strong transcription-regulatory enhancer or promoter chromatin in ostb. These chromatin states had been determined from genome-wide profiles of histone H3 lysine-4 trimethylation (H3K4me3) and $\mathrm{H} 3 \mathrm{~K} 27$ acetylation (H3K27ac) for promoter chromatin and H3K4me1 and H3K27ac for enhancer chromatin. ${ }^{(7)}$ We found that only $~ 0.8 \%$ (440) of the 57,235 index/proxy SNPs were preferentially associated with these types of chromatin in ostb and, in addition, overlapped a narrow peak of H3K27ac preferentially in this cell type (Fig. 1; Supplemental Table S3). Preferential overlap of positive-regulatory chromatin in ostb was defined as such overlap in ostb but in no more than three of the following 12 cell cultures from the Roadmap database ${ }^{(7)}$ (Supplemental Table S3): skin fibroblasts (fib), adult or fetal lung fib, foreskin or dermal melanocytes, keratinocytes, astrocytes, umbilical cord endothelial cells, myoblasts (myob), mammary epithelial cells (HMEC), embryonic stem cells, and a lymphoblastoid cell line. Subsequent filtering for overlap of open chromatin (DHS) in ostb gave 154 


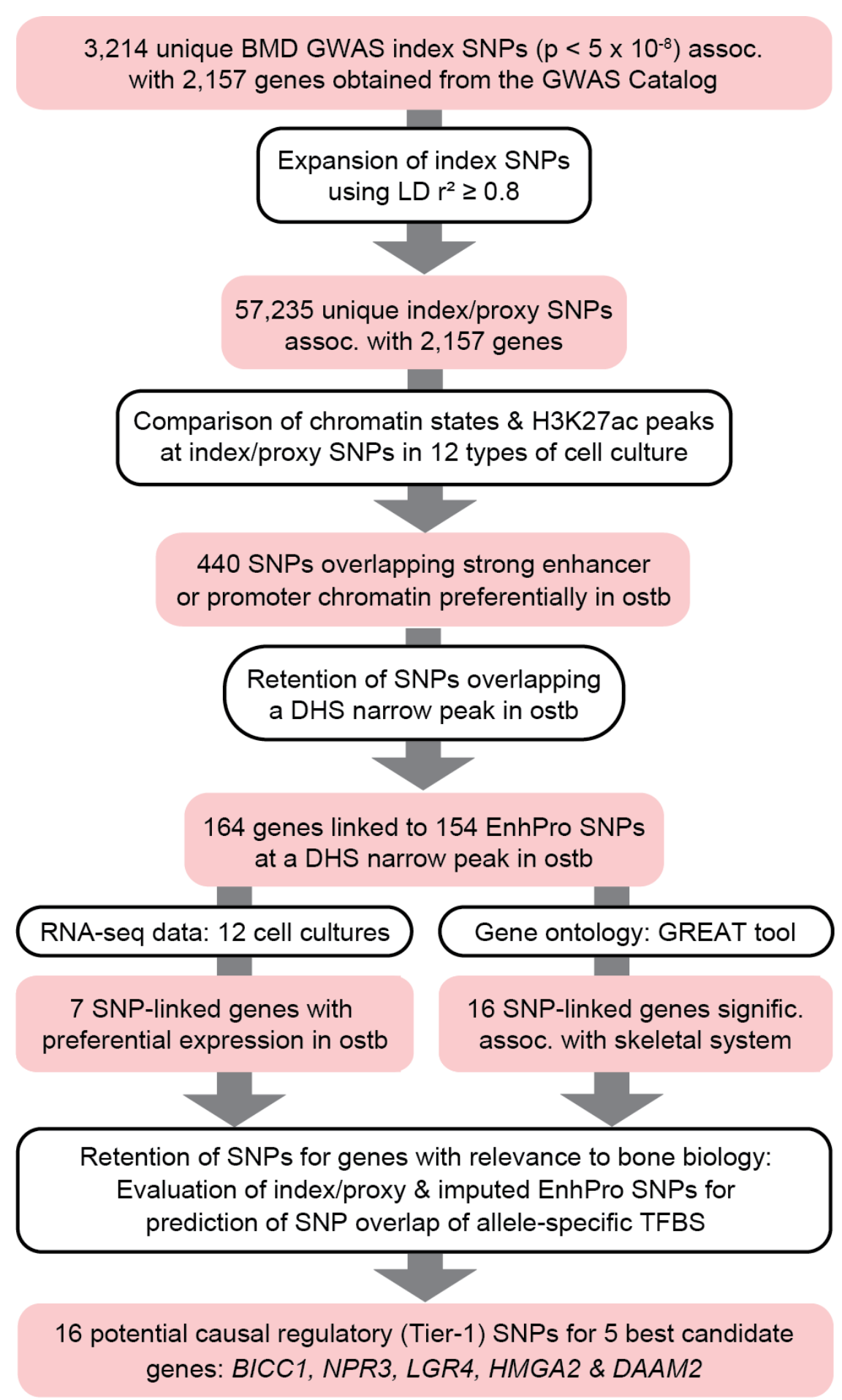

Figure 1. Workflow for prioritizing 16 potential causal regulatory (Tier-1) SNPs from 38 BMD

GWAS. Summary of prioritization of plausible regulatory BMD SNPs. Assoc, associated; H3K27ac, histone H3 lysine-27 acetylation (an epigenetic mark of active enhancers or promoters); ostb, osteoblasts; TFBS, transcription factor binding site; DHS, DNaseI hypersensitive site; GREAT tool, http://great.stanford.edu/public/html/ . 
SNPs, which are referred to as EnhPro SNPs (Supplemental Table S3). EnhPro SNPs often also overlapped strong enhancer or promoter chromatin in bone marrow-derived MSC (74\%) or in chond (79\%) but not in monocytes $(16 \%)$.

In order to identify EnhPro SNPs most likely to contribute to inherited osteoporosis risk, we used two alternate methods to evaluate their associated genes (Fig. 1). First, we identified seven EnhPro SNPassociated genes, $R U N X 2$, TBX15, ADAM12, NPR3, BICC1, LGR4, and SPECC1, that were preferentially expressed in ostb (> 5 times more RPKM in ostb than in the median of 11 heterologous cell types). All but SPECC1 have known bone-related functions. ${ }^{(11,18,27-30)}$ Because we recently analyzed EnhPro SNPs for $T B X 15{ }^{(18)}$ we eliminated it as well as SPECC1 from further consideration. Alternatively, we identified seven additional EnhPro SNP-associated genes, HMGA2, BMP5, DLX6, SIX1, SMAD3, TRPS1, and $W N T 7 B$, that were strongly enriched in bone biology-related Gene Ontology $(\mathrm{GO})$ terms, had literature references for their skeletal association, and displayed appreciable expression in ostb, chond, or MSC (Supplemental Table S5). The EnhPro SNPs from these prioritized genes were examined for predicted overlapping allele-specific TFBS using the TRANSFAC database and stringent hand curation. We found that four of these genes, BICC1, NPR3, LGR4, and HMGA2, had strong predictions of allele-specific TFBS overlapping at least one of their EnhPro SNPs and refer to them as Tier-1 SNPs. The fifth and last Tier-1 SNP-associated gene, DAAM2, was found using a slightly relaxed prioritization procedure for identification of EnhPro SNPs, as described below. In addition to the 13 index/proxy-derived Tier-1 SNPs, we found three more Tier-1 SNPs by examining imputed total body-BMD ${ }^{(3)}$ and eBMD ${ }^{(6)}$ SNPs associated with these five prioritized genes (Table 1).

\section{BICC1 has seven BMD-risk Tier-1 SNPs near the 5' end of the gene}

Among the five prioritized genes, BICCl (BicC Family RNA Binding Protein 1) had the most

Tier-1 SNPs (seven; Table 1). BICC1 encodes a translation-regulatory protein that is implicated in osteoblastogenesis, plays critical roles in several signal transduction pathways, and is required for 
development and homeostasis of various organs including bone. ${ }^{(27,31,32)}$ It is the only gene in the surrounding $1-\mathrm{Mb}$ region that is preferentially and appreciably expressed in ostb and is expressed more strongly in skin fib than in a variety of tissues although more weakly in skin fib than in ostb (Supplemental Figs. S3 and S4). Mouse expression microarray profiles indicate that there is much transcription of Biccl in ostb and negligible expression in osteoclasts, as was the case for all the other Tier-1 SNP associated genes (Supplemental Figs. S5A and B and S6). As positive controls, we checked that osteoclast markers Ctsk, and Tnfrsflla/Rank exhibit the expected osteoclast-associated expression on these microarrays (Supplemental Fig. S5C and D).

The seven BICC1 Tier-1 SNPs were in two distinct clusters starting from $0.1 \mathrm{~kb}$ upstream of the BICC1 transcription start site (TSS - $0.1 \mathrm{~kb}$; rs112597538, rs1896245, and rs1896243) and within the central region of the 107-kb intron 1 (rs1658429, rs11006188, rs1982173, and rs4948523; Fig. 2A and Supplemental Fig. S3E). They were embedded in active promoter chromatin, mixed promoter/enhancer chromatin, or strong enhancer chromatin overlapping DHS in ostb, chond, and MSC (Fig. 2C - E), all of which display high expression of BICC1 (Supplemental Fig. S3). Rs112597538 overlapped a constitutive nucleosome-depleted region seen as a $\sim 0.17-\mathrm{kb}$ hole in the H3K27ac signal surrounding this SNP (triangle, Fig. 2D), which is indicative of strong nucleosome phasing due to TF binding. All seven Tier-1 SNPs are in moderate-to-high LD with each other ( $r^{2}=0.65$ to 1, EUR; Supplemental Fig. S7). Rs112597538, rs1896245, rs1658429 and rs1982173 are located in sequences that are evolutionarily conserved among mammals (Fig. 2B), a trait enriched in non-coding transcription regulatory regions.

Six of the BICC1 Tier-1 SNPs were eQTLs for SkM and tibial artery ( $p=1 \times 10^{-11}$ to $8 \times 10^{-16}$; no data were available for rs112597538 in the GTEx database $\left.{ }^{(23)}\right)$. The eQTLs that were in the proximal region of $B I C C 1$ had positive effect sizes while those in the downstream intron-1 region had negative effect sizes for many tissues including skeletal muscle and aorta $\left(p=2 \times 10^{-4}\right.$ to $\left.3 \times 10^{-8}\right)$ ( $^{(23)}$ Chromatin state profiles make the strongest case for rs112597538 at TSS - $0.1 \mathrm{~kb}$ and rs4948523 at TSS + $66 \mathrm{~kb}$ 
A

B

C

- Str, Wk, Biv Prom

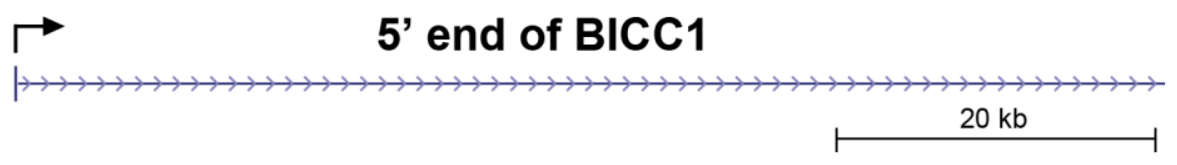

Best regulatory (Tier-1) BMD SNPs (eQTLs colored orange)

rs1896245 rs1896243

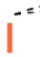

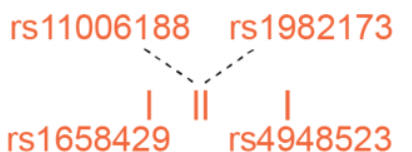

\section{Sequence conservation: Conserved elements in mammals}

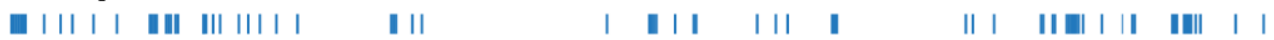

\section{Chromatin state segmentation}

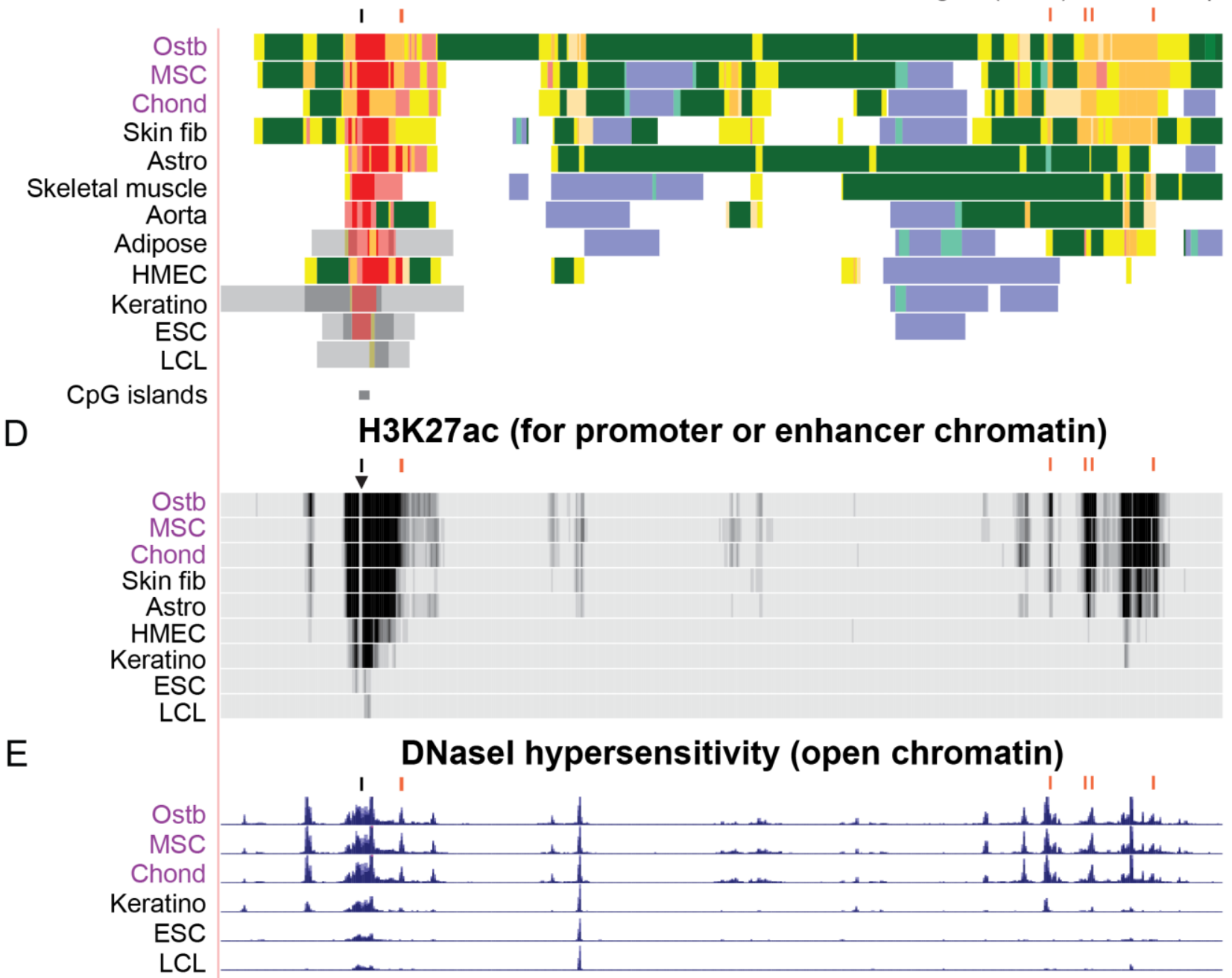

Figure 2. BICC1, which encodes an RNA-binding protein involved in regulating Wnt signaling, has two clusters of Tier-1 SNPs near its 5' end. (A) An 84-kb region around the BICC1 TSS (chr10:60,260,940-60,345,012) with seven Tier-1 SNPs near the TSS (broken arrow) or downstream in intron 1. (B) DNA sequence conservation among placental mammals. $(C)$ Roadmap-derived chromatin state segmentation: strong (Str), moderate (Mod), weak (Wk), or bivalent (Biv; poised) promoter (Prom) or enhancer (Enh) chromatin; repressed (Repr) chromatin; or chromatin with the H3K36me3 mark of 
actively transcribed regions (Txn-chrom); $\mathrm{CpG}$ islands, CpG-rich regions. (D) H327ac enrichment profiles. (E) Profiles of open chromatin (DHS). Short bars above tracks in panels $C, D$, and $E$, positions of Tier-1 SNPs. All tracks were visualized in the UCSC Genome Browser (hg19) and are aligned in this figure and Figs. 3-6. Ostb, osteoblasts; MSC, mesenchymal stem cells; chond, chondrocytes; fib, fibroblasts; astro, astrocytes; HMEC, mammary epithelial cells; NHEK, foreskin keratinocytes; LCL, lymphoblastoid cell line.

(intron 1) driving the eQTL data because these SNPs overlapped promoter or enhancer chromatin in skeletal muscle and aorta, the tissues displaying the BICC1 eQTLs (Fig. 2C). Other Tier-1 SNPs in BICC1 might also influence expression in ostb given the large differences in enhancer chromatin profiles of ostb from those of skeletal muscle or aorta and the lack of available eQTL profiles for ostb.

Importantly, the grouping of BICC1 Tier-1 SNPs according to the directionality of their eQTL is consistent with their grouping by BMD-increasing allele and with their locations in BICC1 (Table 1; Fig. 2). Further support for the biological relevance of several or more of these Tier-1 SNPs to osteoporosis is that TFs predicted to bind in an allele-specific manner to all the BICC1 Tier-1 SNPs were related to the skeletal system (Supplemental Table S6). For example, the Reference genome (Ref) allele, and not the alternate (Alt) allele, of rs112597538 is predicted to bind to SREBP1 and SREBP2 (Table 1), which are implicated in bone biology (Supplemental Table S6).

\section{NPR3 has two intergenic BMD-risk Tier-1 SNPs upstream of a novel lincRNA gene}

There were two Tier-1 SNPs linked to the natriuretic peptide receptor 3 gene (NPR3), which encodes a receptor that facilitates clearance of natriuretic peptides to regulate cell signaling ${ }^{(33)}$ and is implicated in abnormal bone growth phenotypes in humans and mice. ${ }^{(28)} N P R 3$ is moderately to highly expressed in ostb, chond, MSC, lung fib, myob, and skin fib and shows low or negligible expression in the other examined cell cultures (Fig. 3E). Enhancer chromatin profiles paralleled the expression data (Fig. 3C and D). Among various tissues, its highest expression is in aorta (Supplemental Fig. S4B). None 

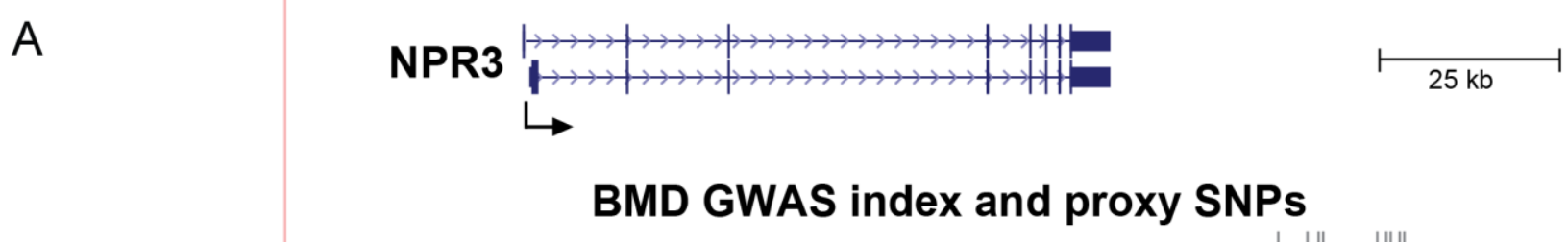

B Best regulatory (Tier-1) BMD SNPs (eQTLs colored orange)
rs $1173771 \mid \quad$ rs 7733331

C

\section{Chromatin state segmentation}

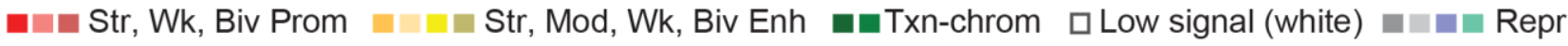

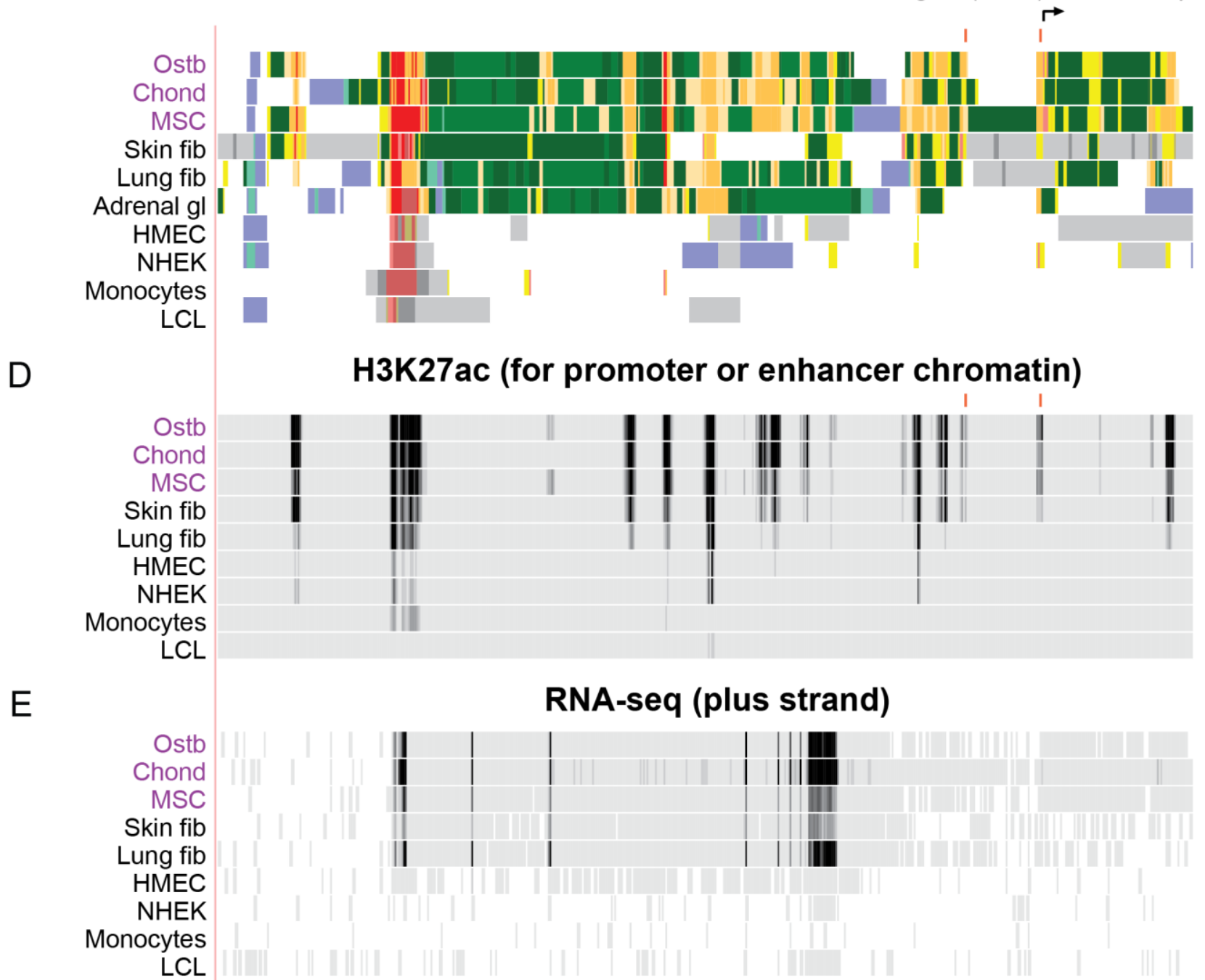

Figure 3. NPR3, which codes for a natriuretic peptide clearance receptor, is associated with two downstream Tier-1 SNPs. (A) NPR3 (chr5:32,678,200-32,856,800) and the BMD GWAS index and proxy SNPs $\left(r^{2} \geq 0.8\right.$, EUR). (B) Tier-1 SNPs. $(C)$ and $(D)$ Chromatin state segmentation and H3K27ac profiles as in Fig. 2. (E) Strand-specific RNA-seq (scale, 0 - 300). Broken arrow in $(C)$, ostb/chond/MSCspecific TSS deduced from the CAGE signal for TSS; see Supplemental Fig. S8). Adrenal gl, adrenal gland; NHEK, skin keratinocytes. 
of the nearby genes within its 1-Mb neighborhood has a transcription profile like that of NPR3 (Supplemental Table S7).

The NPR3 Tier-1 SNPs (rs1173771 and rs7733331) were downstream of the gene, in high LD $\left(r^{2}\right.$ $=0.99)$, and $14 \mathrm{~kb}$ apart (Fig. 3B). Both overlapped nucleosome-depleted subregions of enhancer or mixed enhancer/promoter chromatin seen preferentially in ostb, chond, and MSC (Supplemental Fig. S8D - F). An ostb-, MSC-, and chond-specific TSS for a gene encoding an unannotated long intergenic noncoding RNA (lincRNA) was found only $0.3 \mathrm{~kb}$ from rs7733331 by 5' cap analysis gene expression (CAGE; Fig. 3C, arrow; Supplemental Fig. S8B). This moderate-to-weak RNA-seq signal from the plusstrand was not seen when low-sensitivity settings were used (Fig. 3E) but was seen at higher sensitivity settings specifically in ostb, MSC, and especially chond (Supplemental Fig. S8C). These SNPs were eQTLs for NPR3 $\left(p=4 \times 10^{-41}\right.$ and $\left.6 \times 10^{-39}\right)$ with positive effect sizes in tibial nerve, which moderately expresses this gene (Supplemental Fig. S4B). The trait-decreasing alleles of rs1173771 and rs7733331, and not the opposite alleles, are predicted to bind SATB1 (like BICC1's Tier-1 SNP rs1896245) and GTF2I, respectively (Table 1). Although GTF2I is characterized as a general TF, it binds specifically to initiator and E-box DNA sequence elements in promoters ${ }^{(30)}$ and is implicated in osteoblastogenesis from studies on ostb and mice with heterozygous deletion of the corresponding gene (Supplemental Table S6). Therefore, rs7733331 is an especially attractive candidate for a BMD-regulatory SNP that might affect enhancer-associated NPR3 transcription through a novel lincRNA gene in cis. $^{(34)}$

\section{LGR4 is associated with a BMD-risk Tier-1 SNP downstream of another protein-coding gene}

Leucine rich $\mathrm{G}$ protein-coupled receptor 4 (LGR4/GPR48) was associated with one Tier-1 SNP, rs10835153. This gene encodes a cell membrane receptor that can regulate Wnt signaling and is implicated in both embryonic bone development and postnatal bone remodeling. ${ }^{\left({ }^{35}\right)}$ Rs 10835153 and most of the other BMD-associated SNPs in this region were far downstream of LGR4 and closer to the littlestudied $C C D C 34$ protein-encoding gene and to BBOX1-AS1 than to the LGR4 TSS (Fig. 4C) but neither 


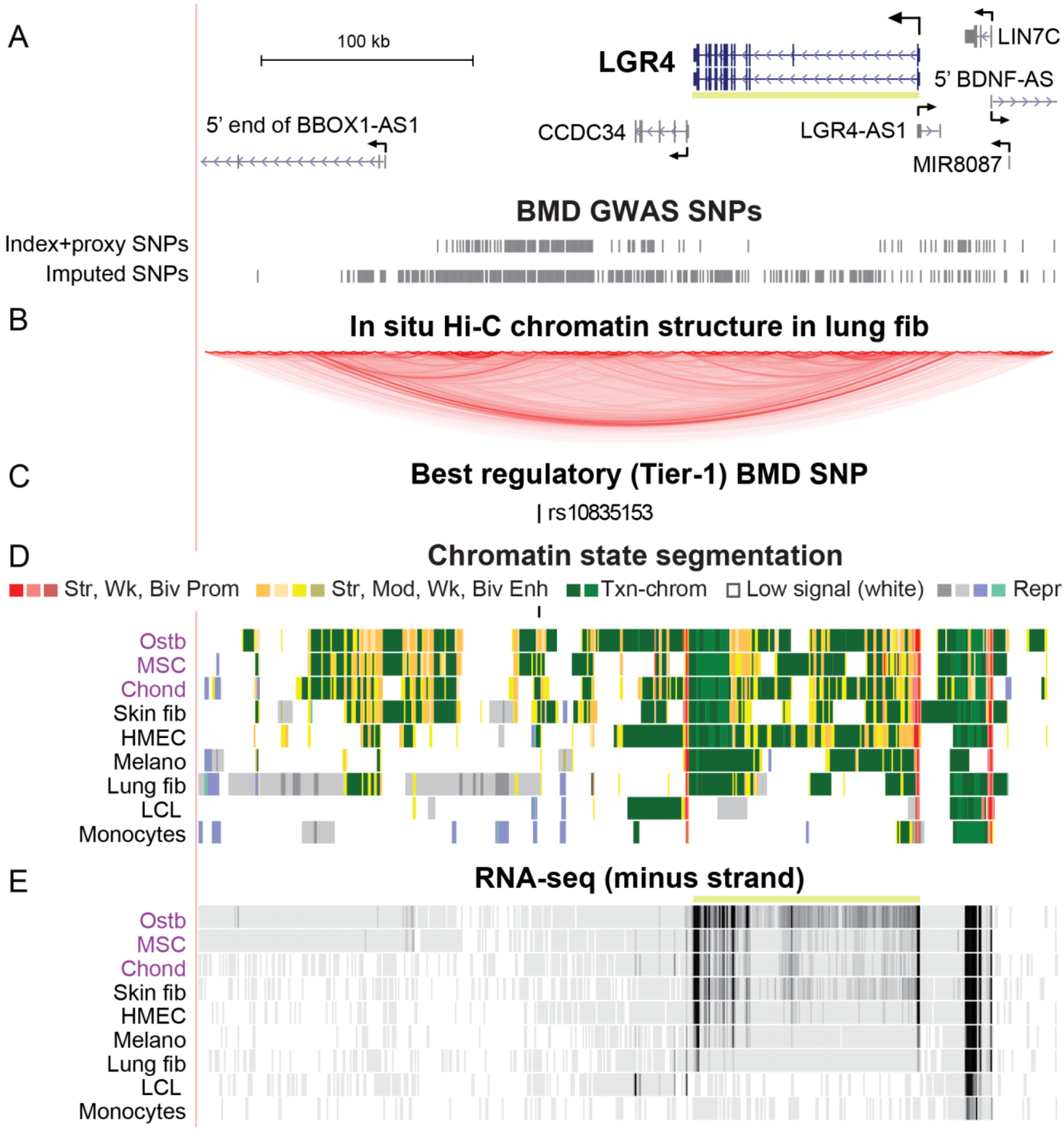

Figure 4. LGR4, which encodes a Wnt signaling receptor, has an intergenic Tier-1 SNP far downstream of the gene. (A) LGR4 and surrounding genes (chr11:27,154,000-27,560,000) and the BMD GWAS index and proxy SNPs as well as imputed SNPs. (B) The Hi-C track ${ }^{(36)}$ for chromatin folding for the fetal lung fib cell line IMR-90; HMEC gave a similar profile (not shown). $(C)-(E)$ as in Fig. 3 except that the minus-strand signal is shown for RNA-seq (scale, $0-100$ ). Melano, foreskin melanocytes. 
of these is appreciably expressed in ostb, MSC, or chond (Supplemental Table S7). Chromatin interaction (Hi-C) profiles ${ }^{(36)}$ for $L G R 4$-expressing lung fib and HMEC indicate that the LGR4 promoter and the ostb enhancer chromatin overlapping LGR4's intergenic Tier-1 SNP, which are $180 \mathrm{~kb}$ apart, are in the same large chromatin loop (topologically associated domain, TAD) ending 2 - 13 kb upstream of the LGR4 TSS in these cells (Fig. 4B). Ostb-specific enhancer chromatin at the Tier-1 SNP and ostb/MSC/chond/HMEC/fib enhancer chromatin further downstream of $L G R 4$ in this TAD are likely to up-regulate the $L G R 4$ promoter because of the similarity in the cell type-specific enhancer chromatin profiles and LGR4 transcription profiles (Fig. 4D and E). The trait-decreasing Alt allele of this Tier-1 SNP is predicted to bind specifically to BPTF, the largest subunit of NURF, a nucleosome remodeling factor. ${ }^{(30)}$ Abnormal phenotypes associated with BPTF in humans and mice include short stature and skeletal abnormalities (Supplemental Table S6).

\section{HMGA2 has three far-upstream BMD-risk Tier-1 SNPs near a novel lincRNA gene}

We identified $H M G A 2$, which encodes high mobility group AT-hook 2 protein, as a prime osteoporosis-risk candidate by GO term enrichment for skeletal system genes rather than by preferential expression in ostb (Fig. 1). HMGA2 is a component of multisubunit enhancer complexes (enhanceosomes). Despite HMGA2's relationship to bone development ${ }^{(37,38)}$ and its ostb-associated superenhancer (a strong, unusually long enhancer) spanning the gene (Fig. 5B, dotted pink line), the RPKM ratio for ostb vs. the median of 11 non-ostb cell cultures other than MSC and chond was only of only 0.3 , and the RPKM for ostb was only 0.5. Even some BMD GWAS-associated genes with negligible expression in the studied human ostb sample were linked to a limb phenotype in mouse models, possibly through effects on osteoclasts, MSC, chond, or on ostb or pre-ostb in vivo but not in vitro (Supplemental Table S8). Similarly, although the steady-state $H M G A 2$ RNA levels were low in the studied ostb, they were high in MSC, which can differentiate into ostb (Fig. 5C; Supplemental Table S7). 
bioRxiv preprint doi: https://doi.org/10.1101/2020.06.18.160309; this version posted June 18, 2020. The copyright holder for this preprint (which was not certified by peer review) is the author/funder, who has granted bioRxiv a license to display the preprint in perpetuity. It is made available under aCC-BY-NC-ND 4.0 International license.

A
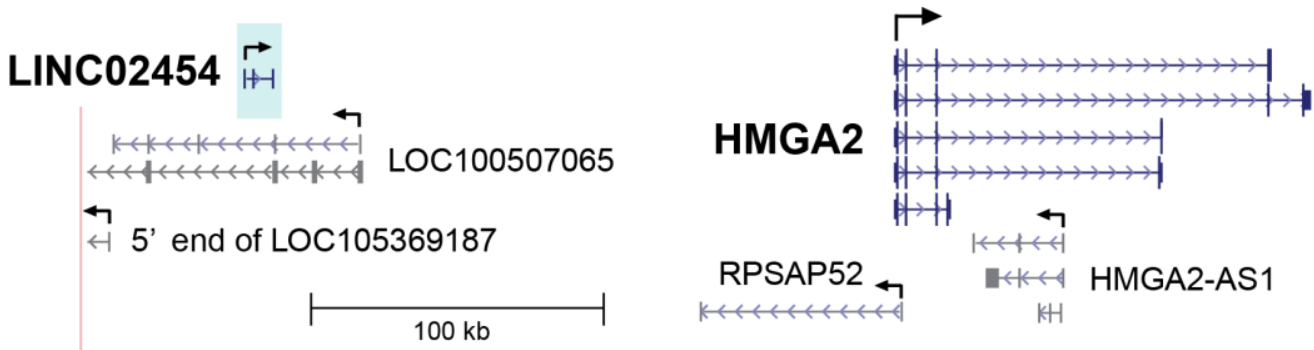

3 SNPs in $615 \mathrm{bp}$

\section{Best regulatory (Tier-1) BMD SNPs}

I rs80019710, rs17101510, rs12296417

B

\section{Chromatin state segmentation}

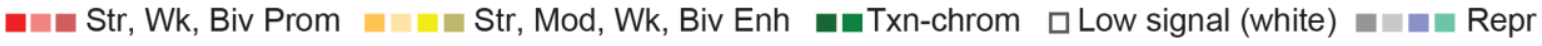

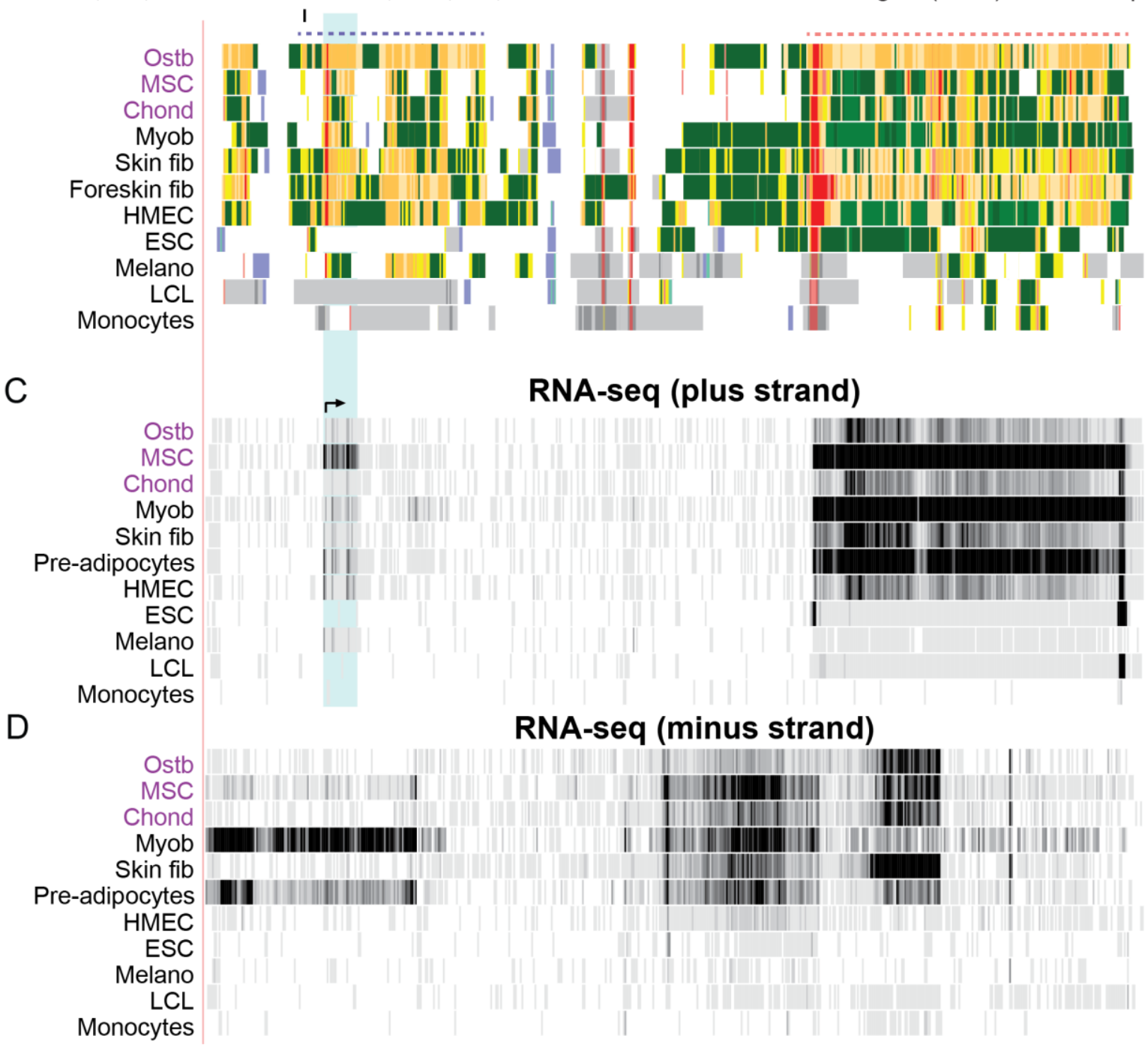

Figure 5. HMGA2, which encodes an enhancesome protein, is associated with a cluster of farupstream intergenic Tier-1 SNPs. (A) HMGA2 and its upstream lincRNA genes and associated Tier-1 SNPs are shown (chr12:65,942,326-66,365,457). $(B)-(D)$ as in previous figures except that the RNA signal from both the plus- and minus-strands is shown (scales, 0 - 90 and 0 - 30, respectively). Dotted lines in panel C indicate super-enhancers in ostb; blue highlighting, LINC02454 region. Myob, myoblasts. 
HMGA2 was associated with three intergenic Tier-1 SNPs (rs80019710, rs17101510, and rs12296417; Fig. 5A) in a 0.6-kb cluster $200 \mathrm{~kb}$ upstream of the HMGA2 TSS. They were embedded in a 85-kb intergenic super-enhancer in ostb and a skin fib cell strain (Fig. 5B, dotted purple line), both of which had low, but appreciable, levels of $H M G A 2$ RNA (Fig. 5C). Within this intergenic super-enhancer are several lincRNA genes, one of which, LINC02454/RP11-221N13.3, is $9 \mathrm{~kb}$ downstream of the clustered Tier-1 SNPs. Its TSS overlaps a constitutive binding site for the looping protein CCCTCbinding factor (CTCF) and is surrounding by ostb/myob/skin fib-associated CTCF sites (Supplemental Fig. S9) that were not seen in cell cultures in which $H M G A 2$ was repressed. This suggests that the subregion containing the Tier-1 SNP cluster is involved in expression-related chromatin looping. Expression patterns of LINCO2454 and several other non-coding RNA (ncRNA) genes in this gene neighborhood were correlated with those of HMGA2 (Fig. 5C and D and Supplemental Fig. S10). One of these ncRNA genes, RPSAP52, can regulate HMGA2 both post-transcriptionally by blocking HMGA2targeted miRNA activity and transcriptionally. ${ }^{(39)}$

From our TFBS analysis, we predict that the three HMGA2 far-upstream Tier-1 SNPs are in DNA sequences that bind HMGA1, YY1, SATB1 or ZBTB20 specifically at their Alt alleles (Table 1). These TFs are associated with bone or cartilage biology (Supplemental Table S6). Of special interest is HMGA1, a chromatin architectural protein similar to HMGA2, which is post-transcriptionally regulated by some of the same miRNAs that control $H M G A 2 \mathrm{RNA}^{(40)}$ and can up-regulate Wnt signaling. ${ }^{(41)}$ Moreover, HMGA1 is critical for forming and maintaining bone and downregulates $m i R-196 A-2$, which targets $H M G A 2$ RNA in mouse embryonic fib. ${ }^{(42)}$

\section{Slightly varying search parameters for BMD-risk SNPs revealed likely osteoporosis-risk regulatory SNPs for DAAM2}

By slightly relaxing our standard protocol for identifying Tier-1 SNPs, we identified three clustered SNPs (rs2504105, rs2504104, and rs2504103) associated with DAAM2 (Dishevelled associated 
activator of morphogenesis 2) as highly credible candidates for BMD-associated regulatory SNPs (Fig. 6). In this modified protocol, we allowed BMD GWAS-derived EnhPro SNPs to overlap a H3K27ac narrow peak in ostb and in no more than four (rather than the previous three) of the 12 other examined cell cultures from the Roadmap database but retained all the other requirements for Tier-1 SNPs (Fig. 1). $D A A M 2$ encodes a key regulator of the Wnt signaling pathway and is implicated in ostb mineralization as well as in bone resorption by murine osteoclasts. ${ }^{(6,43)}$ It has a ratio of 21 for ostb/non-ostb RNA levels (Supplementary Table S2) although its expression in chond and skin fib is higher than in ostb (Fig. 6E).

Only one eBMD index SNP (rs2504101) was reported for DAAM2 in a study by Morris et al., ${ }^{(6)}$ who featured this gene as a candidate causal gene for osteoporosis risk. We found that their selected index SNP in intron 2 of DAAM2 (Fig. 6A, triangle) overlaps transcription-type (H3K36me3-enriched) chromatin and not enhancer or promoter chromatin in ostb, MSC, chond, and skin fib and does not overlap a DHS in any examined cell type. From 38 SNPs in high LD with this index SNP $\left(r^{2}>0.8\right.$, EUR; Supplemental Table S3), we identified three (rs2504105, rs2504104, and rs2504103; LD $\left.r^{2}=0.97\right)$ as Tier-1 SNPs using the above-described criterion for H3K27ac specificity. These SNPs span only 27 bp and were embedded in mixed enhancer/promoter chromatin in ostb and enhancer chromatin or enhancer/promoter chromatin in MSC, chond, and skin fib. They were located $0.8 \mathrm{~kb}$ upstream of exon 2 of DAAM2 and $0.4 \mathrm{~kb}$ upstream of a novel sense-strand intronic TSS (CAGE signal; Fig. 6C, arrow) in these cell types but not in cell cultures with repressed HMGA2. Moreover, they are eQTLs for tibial nerve $\left(p=8.4 \times 10^{-6}\right)$, a DAAM2-expressing tissue (Supplemental Fig. S4D). These findings suggest that the cluster of Tier-1 SNPs up-regulates DAAM2 expression partly by upregulating expression of an intronic ncRNA transcript at a cell type-specific enhancer. The much lower steady-state levels of DAAM2 RNA in ostb and MSC than in chond despite their similar amounts of enhancer chromatin could be due to posttranscriptional regulation. 
A

B

C
DAAM2

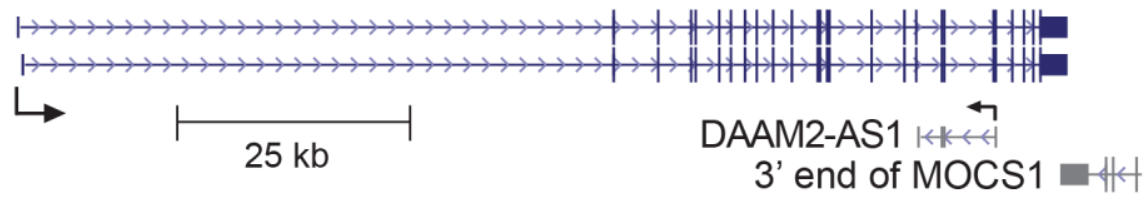

BMD GWAS index SNP (triangle) and its proxies

| | || || | | || || |||| | || | |||| | |

\section{Best regulatory (Tier-1) BMD SNPs (eQTLs colored orange)}

3 SNPs in 27 bp | rs2504104

\section{Chromatin state segmentation}

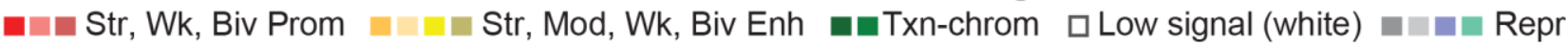

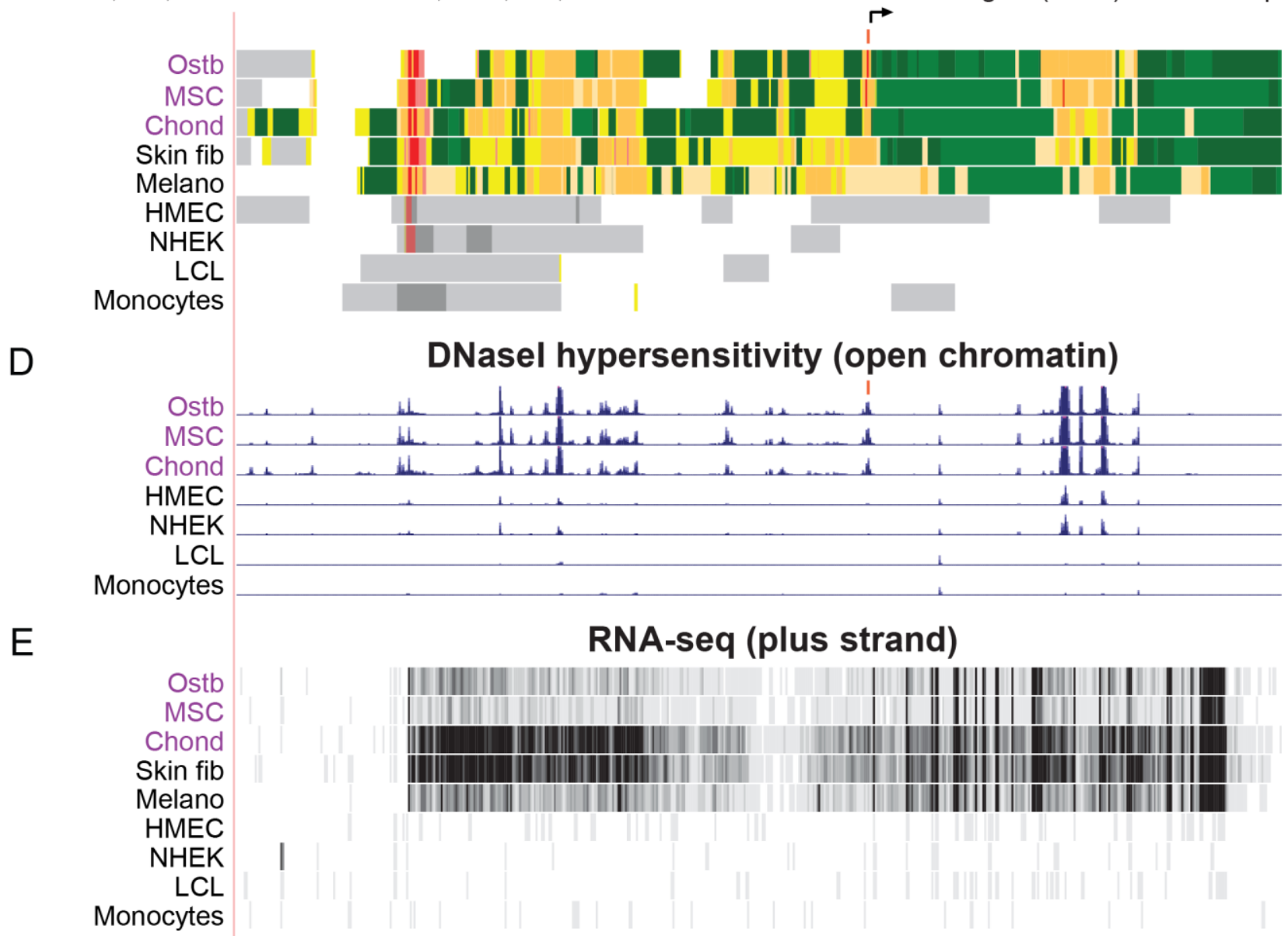

Figure 6. DAAM2, which codes for a Wnt signaling protein, has a cluster of three intragenic Tier-1 SNPs. (A) DAAM2 and its index and proxy SNPs from BMD GWAS (chr6:39,735,841-39,911,256). (B) $-(E)$ as in previous figures except that the scale for RNA-seq was 0-50; NHEK, skin keratinocytes. 
Like rs11006188 in BICC1, the Ref allele of DAAM2 Tier-1 SNP rs2504105 is predicted to bind SMAD family TFs (Supplemental Table S6). In addition, the Alt allele of Tier-1 SNP rs2504104 overlaps predicted binding sites for RUNX2, the master regulator of ostb lineage commitment, ${ }^{(11)}$ and CBFB, which can help stabilize Runx-family proteins binding to $\mathrm{DNA}^{(44)}$ (Table 1). Lastly, the Alt allele of rs2504103 is predicted to bind NFIC, which plays important roles in both tooth and bone development. ${ }^{(45)}$ These findings support the candidacy of one or more of these SNPs as causal regulatory SNPs for BMD risk.

\section{Discussion}

We identified 16 high-priority osteoporosis-risk regulatory SNPs by using an unusual in-depth bioinformatic approach relying on epigenomic and transcriptomic comparisons of ostb and heterologous cell cultures as well as prediction of allele-specific binding of TFs that are important for bone biology. These Tier-1 SNPs, which had not been previously identified as candidates for causal regulatory SNPs for $\mathrm{BMD}$, were associated with five skeletal system-related genes. Three of these, BICC1, LGR4, DAAM2, are involved directly or indirectly in canonical Wnt signaling, a signaling pathway that plays critical roles in osteoblastogenesis and bone homeostasis. ${ }^{(46-48)}$ The other two genes are NPR3, and HMGA2, a mediator of natriuretic signaling and an essential component of enhanceosomes, respectively. ${ }^{(28,49)}$

One of the most interesting genes for which we identified novel regulatory candidate SNPs is $B I C C 1$, which encodes a self-polymerizing RNA-binding protein that indirectly regulates Wnt signaling. ${ }^{(32)}$ This gene had two clusters of Tier-1 SNPs near its 5' end (Fig. 2). BICC1 is involved in osteoblastogenesis and polycystic kidney disease, in part, through its inhibition of post-transcriptional silencing of $P K D 2$ RNA by $m i R-17 .{ }^{(31,50,51)} \mathrm{PKD} 2 / \mathrm{PC} 2$ and PKD1/PC1 form primary cilia that function as osteoporosis-related mechanosensors in ostb (and probably osteocytes) and in epithelial kidney cells, 
and BICC1 has been detected in primary cilia in kidney cells. ${ }^{(32,52-54)}$ Conditional inactivation of $P k d 2$ in ostb/osteocytes of mice results in decreased levels of Runx2 RNA and Wnt signaling along with the development of osteopenia. ${ }^{(51)}$ An important study by Mesner et al. ${ }^{(31)}$ implicating BICC1 in osteoporosis demonstrated that a heterozygous inactivating mutation in Biccl in male mice led to low femoral BMD and that Biccl-deficent ostb had impaired differentiation in vitro that could be rescued by $P k d 2$ overexpression.

LGR4, which had one associated Tier-1 SNP (Fig. 4), encodes a bone-related Wnt signaling receptor $^{(29,55)}$ and had been catalogued as one of many genes with an eBMD-associated missense SNPs (rs34804482) predicted to have a deleterious effect on protein structure. ${ }^{(6)}$ Moreover, Styrkarsdottir et al. ${ }^{(56)}$ found a rare nonsense mutation in the gene that is associated with low BMD, osteoporotic fractures in elderly individuals, electrolyte imbalance, and several types of cancer. In mice, homozygous deletion of Lgr4 results in a low-BMD phenotype, a strong delay in ostb differentiation and mineralization, and elevated numbers of osteoclasts. ${ }^{(29)}$ Osteoclasts and MSC precursors of ostb have also been implicated in the positive effects of LGR4 on bone formation and homeostasis from conditional mouse knock-out models and in vitro studies. ${ }^{(57,58)}$ However, the single Tier-1 SNP that we found to be associated with LGR4 was in enhancer chromatin in ostb but not in MSC. Epigenetic profiles of osteoclasts are not available but the finding of negligible levels of expression of Lgr4 (and the mouse homologs of the other Tier-1 associated genes) in cultured mouse osteoclasts and substantial levels in mouse ostb (Supplemental Fig. S6) suggests that the LGR4 Tier-1 SNP's regulatory role in osteoporosis risk is through ostb and possibly osteocytes.

Like $L G R 4, D A A M 2$ encodes a regulator of canonical Wnt signaling implicated in the ostb and osteoclast lineages. $^{(6,43,59)}$ Its 27-bp cluster of three intragenic Tier-1 SNPs, which are in perfect LD, may act through their overlapping enhancer/promoter chromatin in ostb, MSC, and chond and the novel intragenic TSS immediately downstream of these SNPs (Fig. 6C, arrow). Consistent with a role for 
$D A A M 2$ in inherited osteoporosis risk, an eBMD GWAS-derived missense variant (rs201229319) in $D A A M 2$ that is probably deleterious had been identified previously by Morris et al. ${ }^{(6)}$ They also found that homozygous knockout of Daam2 in mice decreases bone strength and increases cortical bone porosity and that CRISPR/Cas9-mediated inactivation of DAAM2 impairs mineralization in an ostb cell line. We identified the DAAM2 Tier-1 SNPs by a very small change in one of our requirements for Tier-1 SNP designation (Fig. 1) to $<5$ rather than $<4$ of the 12 non-ostb related cell cultures displaying the ostbassociated H3K27ac peak overlapping the SNP. Therefore, future bioinformatic studies could uncover many more plausible BMD-regulatory SNPs by small variations of our protocol.

NPR3 (NPRC), one of the Tier-1 SNP associated genes, is involved not only in linear bone growth, bone turnover, and endochondral ossification, ${ }^{(28,60-62)}$ but also in cardiovascular homeostasis, and renal cancer metastasis. ${ }^{(28,62)}$ Its Tier-1 SNPs (rs1173771 and rs7733331), which are in almost perfect LD, are upstream of another novel TSS observed preferentially in ostb and chond (Fig. 3C and Supplemental Fig. S8). NPR3 encodes a transmembrane clearance receptor that opposes natriuretic peptide signaling through NPR1 and NPR2 receptors, which, unlike NPR3, contain guanylyl cyclase activity that is activated upon peptide binding. Enhancement of bone growth was found in patients with bi-allelic NPR3 loss-of-function mutations or monoallelic gain-of-function mutations in NPR2. ${ }^{(28)}$ Osteocrin, a protein structurally resembling natriuretic peptides, binds specifically to NPR3 and is implicated in stimulating bone growth by limiting NPR3-mediated clearance of natriuretic peptides. ${ }^{(63)}$ NPR3 was associated with high bone density in a GWAS. ${ }^{(64)} N P R 3$ is downregulated post-transcriptionally by $m i R-143,{ }^{(65)}$ whose host gene, CARMN, displays especially high expression in ostb. ${ }^{(66)}$ Both NPR3-associated Tier-1 SNPs have been previously identified as index SNPs in many blood pressure-related GWAS, consistent with the role of NPR3 also in the cardiovascular system. ${ }^{(19)}$ In addition, rs1173771 was associated with height in a GWAS. ${ }^{(67)}$ 
HMGA2, which has three clustered Tier-1 SNPs far upstream of the gene (Fig. 5), was the only one of the five prioritized genes that was identified by its bone-related GO association rather than by its ostb/non-ostb expression ratio (Fig. 1). Some genes that impact osteoporosis risk through the ostb lineage may act at the pre-ostb or osteocyte stages. Alternatively, some osteoporosis-associated genes might be negatively associated with osteoblastogenesis. $H M G A 2$ may be an example of both phenomena. It encodes a chromosomal architectural and enhanceosomal protein implicated in negatively regulating the differentiation of bone marrow-derived MSC to ostb ${ }^{(68)}$ and is necessary for normal embryogenesis, including bone development. ${ }^{(37,38)}$ It also is involved in carcinogenesis and metastasis, ${ }^{(69)}$ cell replication, and autophagy, which plays central roles in bone homeostasis through effects on ostb, osteocytes, and osteoclasts. ${ }^{(70)} H M G A 2$ displays very low or negligible expression in normal postnatal tissues but is highly expressed in some mesoderm-derived stem or progenitor cell types including MSC, myob, and preadipocytes. It is much more weakly expressed in ostb, in accord with its negative effects on ostb formation from MSC. ${ }^{(49)}$ The need for tight control of $H M G A 2$ expression is evidenced by its unusually complex regulatory circuitry including the cancer-associated let-7 miRNA and the HMGA2-overlapping pseudogene RPSAP52 and anti-sense gene HMGA2-AS1. ${ }^{(49,71)}$ Importantly, another ncRNA gene, LINC02454, which exhibits an HMGA2-like expression pattern (Supplemental Fig. S10), is $9 \mathrm{~kb}$ downstream of the three Tier-1 SNPs in an ostb-associated super-enhancer far from HMGA2. LINC02454 was previously referenced only for its dysregulation in cancer cells. ${ }^{(72)} \mathrm{We}$ propose that one or several of these Tier-1 SNPs help control expression of LINC02454, which, in turn, helps regulate transcription of HMGA2 in MSC. The much lower steady-state levels of HMGA2 mRNA in ostb than in MSC despite the abundant enhancer chromatin within or upstream of the gene in ostb (Fig. 5) may be due to multiple miRNAs targeting HMGA2. ${ }^{(71)}$ Besides a role for $H M G A 2$ in negatively regulating MSC differentiation to ostb, it may play a protective role in the skeleton ${ }^{(73)}$ at later steps in differentiation of ostb to osteocytes and in osteocyte homeostasis by helping to induce autophagy. ${ }^{(70,74,75)}$ 
Like our five osteoporosis-risk candidate genes, the TFs predicted to bind to the Tier-1 SNPcontaining DNA sequences associated with these genes have special functions in the skeletal system (Supplemental Table S6). Further evidence implicating Tier-1 SNPs as transcription-regulatory variants comes from our finding that 11 of the 16 SNPs for BICC1, DAAM2, or NPR3 overlapped eQTLs for one or several tissues in which these genes are expressed. However, eQTLs are not available for ostb and, given our requirement that Tier-1 SNPs be present in enhancer or promoter chromatin preferentially in ostb, eQTL overlap of Tier-1 SNPs should be underrepresented by available data. In the cases of NPR3, and DAAM2, which had 2 - 3 Tier-1 SNPs in high LD, only a single SNP at each of these loci may be the regulatory causal SNP. However, for BICC1, the dichotomy of the direction of effect sizes for tissue eQTLs and the distribution of BMD-increasing alleles for the two clusters of Tier-1 SNPs suggests that at least one Tier-1 SNP in the TSS-adjacent cluster and one in the 60-kb downstream cluster are causal regulatory SNPs.

\section{Conclusion and future directions}

Candidates for highly credible causal regulatory GWAS-derived variants are more difficult to prioritize but are much more numerous than exonic variants affecting polypeptide structure. For our selection of 16 osteoporosis-related GWAS regulatory SNPs associated with five genes from 38 BMD GWAS, we used very restrictive epigenetic and transcriptomic criteria. The approach we took to prioritize these osteoporosis-associated genes and their Tier-1 SNPs for future experimental studies can be extended for additional prioritization of candidates for regulatory BMD SNPs, for example, by changing the threshold for preferential expression in ostb, using different tools for gene functional analysis, making small changes in the requirements for designation of preferential enhancer or promoter chromatin in ostb, looking for preferential expression in chond instead of ostb, and expanding the analyses of epigenomic and transcriptomic profiles to osteoclasts and osteocytes as these profiles become available. Elucidation of regulatory genetic variants strongly associated with disease-susceptibility using epigenetic-intensive 
strategies like ours can help find marker SNPs to identify at-risk individuals for treatment or life-style modifications and to develop pharmacological interventions by focusing attention on insufficiently studied protein-coding genes and neighboring ncRNA genes.

\section{Disclosures}

All of the authors state that they have no conflict of interest.

\section{Acknowledgements}

This study was partially supported or benefited by grants from the National Institutes of Health [P20GM109036, R01AR069055, U19AG055373, and R01MH104680], and the Edward G. Schlieder

Endowment and Drs. W. C. Tsai and P. T. Kung Professorship in Biostatistics from Tulane University.

\section{Authors contributions}

XZ and ME designed the study. H-WD and HS gave advice on the study design. XZ performed analysis of the data and prepared figures and tables. ME and XZ wrote the manuscript. All authors revised and reviewed the paper. 


\section{References}

1. Al-Barghouthi BM, Farber CR. Dissecting the Genetics of Osteoporosis using Systems Approaches. Trends Genet. 2019;35(1):55-67.

2. Manolagas SC. Birth and death of bone cells: basic regulatory mechanisms and implications for the pathogenesis and treatment of osteoporosis. Endocr Rev. 2000;21(2):115-37.

3. Medina-Gomez C, Kemp JP, Trajanoska K, Luan J, Chesi A, Ahluwalia TS, et al. Life-Course Genome-wide Association Study Meta-analysis of Total Body BMD and Assessment of Age-Specific Effects. Am J Hum Genet. 2018;102(1):88-102.

4. Kim SK. Identification of 613 new loci associated with heel bone mineral density and a polygenic risk score for bone mineral density, osteoporosis and fracture. PLoS One. 2018;13(7):e0200785.

5. Zheng HF, Forgetta V, Hsu YH, Estrada K, Rosello-Diez A, Leo PJ, et al. Whole-genome sequencing identifies EN1 as a determinant of bone density and fracture. Nature. 2015;526(7571):112-7. 6. Morris JA, Kemp JP, Youlten SE, Laurent L, Logan JG, Chai RC, et al. An atlas of genetic influences on osteoporosis in humans and mice. Nat Genet. 2019;51(2):258-66.

7. Kundaje A, Meuleman W, Ernst J, Bilenky M, Yen A, Heravi-Moussavi A, et al. Integrative analysis of 111 reference human epigenomes. Nature. 2015;518(7539):317-30.

8. Kemp JP, Morris JA, Medina-Gomez C, Forgetta V, Warrington NM, Youlten SE, et al. Identification of 153 new loci associated with heel bone mineral density and functional involvement of GPC6 in osteoporosis. Nat Genet. 2017;49(10):1468-75.

9. Kichaev G, Yang WY, Lindstrom S, Hormozdiari F, Eskin E, Price AL, et al. Integrating functional data to prioritize causal variants in statistical fine-mapping studies. PLoS Genet.

2014;10(10):e1004722.

10. Tak YG, Farnham PJ. Making sense of GWAS: using epigenomics and genome engineering to understand the functional relevance of SNPs in non-coding regions of the human genome. Epigenetics Chromatin. 2015;8:57.

11. Allas L, Boumediene K, Bauge C. Epigenetic dynamic during endochondral ossification and articular cartilage development. Bone. 2019;120:523-32.

12. Husain A, Jeffries MA. Epigenetics and Bone Remodeling. Curr Osteoporos Rep. 2017;15(5):4508.

13. Ono N, Balani DH, Kronenberg HM. Stem and progenitor cells in skeletal development. Curr Top Dev Biol. 2019;133:1-24.

14. Yang L, Tsang KY, Tang HC, Chan D, Cheah KS. Hypertrophic chondrocytes can become osteoblasts and osteocytes in endochondral bone formation. Proc Natl Acad Sci U S A.

2014;111(33):12097-102.

15. Wolff LI, Hartmann C. A Second Career for Chondrocytes-Transformation into Osteoblasts. Curr Osteoporos Rep. 2019;17(3):129-37.

16. Maeda J, Saiki K, Kondo H, Matsuo H, Imamura T, Ogami-Takamura K, et al. Diversity in intracortical remodeling in the human femoral bone: A novel view point with the morphological analysis of secondary osteons. J Orthop Sci. 2018;23(6):1079-86.

17. Intemann J, De Gorter DJJ, Naylor AJ, Dankbar B, Wehmeyer C. Importance of osteocytemediated regulation of bone remodelling in inflammatory bone disease. Swiss Med Wkly. 2020;150:w20187.

18. Zhang X, Ehrlich KC, Yu F, Hu X, Meng XH, Deng HW, et al. Osteoporosis- and obesity-risk interrelationships: an epigenetic analysis of GWAS-derived SNPs at the developmental gene TBX15. Epigenetics. 2020;15(6-7):728-49.

19. Buniello A, MacArthur JAL, Cerezo M, Harris LW, Hayhurst J, Malangone C, et al. The NHGRIEBI GWAS Catalog of published genome-wide association studies, targeted arrays and summary statistics 2019. Nucleic Acids Res. 2019;47(D1):D1005-D12. 
20. Genomes Project C, Auton A, Brooks LD, Durbin RM, Garrison EP, Kang HM, et al. A global reference for human genetic variation. Nature. 2015;526(7571):68-74.

21. Purcell S, Neale B, Todd-Brown K, Thomas L, Ferreira MA, Bender D, et al. PLINK: a tool set for whole-genome association and population-based linkage analyses. Am J Hum Genet. 2007;81(3):559-

75.

22. Machiela MJ, Chanock SJ. LDlink: a web-based application for exploring population-specific haplotype structure and linking correlated alleles of possible functional variants. Bioinformatics. 2015;31(21):3555-7.

23. Consortium GT. Human genomics. The Genotype-Tissue Expression (GTEx) pilot analysis: multitissue gene regulation in humans. Science. 2015;348(6235):648-60.

24. Wu C, Orozco C, Boyer J, Leglise M, Goodale J, Batalov S, et al. BioGPS: an extensible and customizable portal for querying and organizing gene annotation resources. Genome Biol. 2009;10(11):R130.

25. Huang da W, Sherman BT, Lempicki RA. Systematic and integrative analysis of large gene lists using DAVID bioinformatics resources. Nat Protoc. 2009;4(1):44-57.

26. McLean CY, Bristor D, Hiller M, Clarke SL, Schaar BT, Lowe CB, et al. GREAT improves functional interpretation of cis-regulatory regions. Nat Biotechnol. 2010;28(5):495-501.

27. Baron R, Kneissel M. WNT signaling in bone homeostasis and disease: from human mutations to treatments. Nat Med. 2013;19(2):179-92.

28. Boudin E, de Jong TR, Prickett TCR, Lapauw B, Toye K, Van Hoof V, et al. Bi-allelic Loss-ofFunction Mutations in the NPR-C Receptor Result in Enhanced Growth and Connective Tissue Abnormalities. Am J Hum Genet. 2018;103(2):288-95.

29. Luo J, Zhou W, Zhou X, Li D, Weng J, Yi Z, et al. Regulation of bone formation and remodeling by G-protein-coupled receptor 48. Development. 2009;136(16):2747-56.

30. Stelzer G, Rosen N, Plaschkes I, Zimmerman S, Twik M, Fishilevich S, et al. The GeneCards Suite: From Gene Data Mining to Disease Genome Sequence Analyses. Curr Protoc Bioinformatics. 2016;54:130 1-1 3.

31. Mesner LD, Ray B, Hsu YH, Manichaikul A, Lum E, Bryda EC, et al. Bicc1 is a genetic determinant of osteoblastogenesis and bone mineral density. J Clin Invest. 2014;124(6):2736-49.

32. Rothe B, Gagnieux C, Leal-Esteban LC, Constam DB. Role of the RNA-binding protein BicaudalC1 and interacting factors in cystic kidney diseases. Cell Signal. 2020;68:109499.

33. Almeida FA, Suzuki M, Scarborough RM, Lewicki JA, Maack T. Clearance function of type C receptors of atrial natriuretic factor in rats. Am J Physiol. 1989;256(2 Pt 2):R469-75.

34. Lewis MW, Li S, Franco HL. Transcriptional control by enhancers and enhancer RNAs.

Transcription. 2019;10(4-5):171-86.

35. Luo J, Sun P, Siwko S, Liu M, Xiao J. The role of GPCRs in bone diseases and dysfunctions. Bone Res. 2019;7:19.

36. Rao SS, Huntley MH, Durand NC, Stamenova EK, Bochkov ID, Robinson JT, et al. A 3D map of the human genome at kilobase resolution reveals principles of chromatin looping. Cell.

2014;159(7):1665-80.

37. Ligon AH, Moore SD, Parisi MA, Mealiffe ME, Harris DJ, Ferguson HL, et al. Constitutional rearrangement of the architectural factor HMGA2: a novel human phenotype including overgrowth and lipomas. Am J Hum Genet. 2005;76(2):340-8.

38. Zhou X, Benson KF, Ashar HR, Chada K. Mutation responsible for the mouse pygmy phenotype in the developmentally regulated factor HMGI-C. Nature. 1995;376(6543):771-4.

39. Ros G, Pegoraro S, De Angelis P, Sgarra R, Zucchelli S, Gustincich S, et al. HMGA2 Antisense Long Non-coding RNAs as New Players in the Regulation of HMGA2 Expression and Pancreatic Cancer Promotion. Front Oncol. 2019;9:1526. 
40. Tian X, Song J, Zhang X, Yan M, Wang S, Wang Y, et al. MYC-regulated pseudogene HMGA1P6 promotes ovarian cancer malignancy via augmenting the oncogenic HMGA1/2. Cell Death Dis. 2020;11(3):167.

41. Xian L, Georgess D, Huso T, Cope L, Belton A, Chang YT, et al. HMGA1 amplifies Wnt signalling and expands the intestinal stem cell compartment and Paneth cell niche. Nat Commun. 2017;8:15008.

42. De Martino I, Visone R, Fedele M, Petrocca F, Palmieri D, Hoyos JM, et al. Regulation of microRNA expression by HMGA1 proteins. Oncogene. 2016;35(44):5817-8.

43. Uehara S, Udagawa N, Mukai H, Ishihara A, Maeda K, Yamashita T, et al. Protein kinase N3 promotes bone resorption by osteoclasts in response to Wnt5a-Ror2 signaling. Sci Signal. 2017;10(494).

44. Qin X, Jiang Q, Matsuo Y, Kawane T, Komori H, Moriishi T, et al. Cbfb regulates bone development by stabilizing Runx family proteins. J Bone Miner Res. 2015;30(4):706-14.

45. Roh SY, Park JC. The role of nuclear factor I-C in tooth and bone development. J Korean Assoc Oral Maxillofac Surg. 2017;43(2):63-9.

46. Maeda K, Kobayashi Y, Koide M, Uehara S, Okamoto M, Ishihara A, et al. The Regulation of Bone Metabolism and Disorders by Wnt Signaling. Int J Mol Sci. 2019;20(22).

47. Houschyar KS, Tapking C, Borrelli MR, Popp D, Duscher D, Maan ZN, et al. Wnt Pathway in Bone Repair and Regeneration - What Do We Know So Far. Front Cell Dev Biol. 2018;6:170.

48. Kobayashi Y, Uehara S, Udagawa N. Roles of non-canonical Wnt signaling pathways in bone resorption. Journal of Oral Biosciences. 2018;60(2):31-5.

49. Parisi S, Piscitelli S, Passaro F, Russo T. HMGA Proteins in Stemness and Differentiation of Embryonic and Adult Stem Cells. Int J Mol Sci. 2020;21(1).

50. Tran U, Zakin L, Schweickert A, Agrawal R, Doger R, Blum M, et al. The RNA-binding protein bicaudal $\mathrm{C}$ regulates polycystin 2 in the kidney by antagonizing miR-17 activity. Development. 2010;137(7):1107-16.

51. Xiao Z, Cao L, Liang Y, Huang J, Stern AR, Dallas M, et al. Osteoblast-specific deletion of Pkd2 leads to low-turnover osteopenia and reduced bone marrow adiposity. PLoS One. 2014;9(12):e114198.

52. Hoey DA, Chen JC, Jacobs CR. The primary cilium as a novel extracellular sensor in bone. Front Endocrinol (Lausanne). 2012;3:75.

53. Mohieldin AM, Haymour HS, Lo ST, AbouAlaiwi WA, Atkinson KF, Ward CJ, et al. Protein composition and movements of membrane swellings associated with primary cilia. Cell Mol Life Sci. 2015;72(12):2415-29.

54. Xiao Z, Zhang S, Cao L, Qiu N, David V, Quarles LD. Conditional disruption of Pkd1 in osteoblasts results in osteopenia due to direct impairment of bone formation. J Biol Chem. 2010;285(2):1177-87.

55. Zhu C, Zheng XF, Yang YH, Li B, Wang YR, Jiang SD, et al. LGR4 acts as a key receptor for Rspondin 2 to promote osteogenesis through Wnt signaling pathway. Cell Signal. 2016;28(8):989-1000.

56. Styrkarsdottir U, Thorleifsson G, Sulem P, Gudbjartsson DF, Sigurdsson A, Jonasdottir A, et al. Nonsense mutation in the LGR4 gene is associated with several human diseases and other traits. Nature. 2013;497(7450):517-20.

57. Luo J, Yang Z, Ma Y, Yue Z, Lin H, Qu G, et al. LGR4 is a receptor for RANKL and negatively regulates osteoclast differentiation and bone resorption. Nat Med. 2016;22(5):539-46.

58. Sun P, Jia K, Zheng C, Zhu X, Li J, He L, et al. Loss of Lgr4 inhibits differentiation, migration and apoptosis, and promotes proliferation in bone mesenchymal stem cells. J Cell Physiol. 2019;234(7):10855-67.

59. Lee HK, Chaboub LS, Zhu W, Zollinger D, Rasband MN, Fancy SP, et al. Daam2-PIP5K is a regulatory pathway for Wnt signaling and therapeutic target for remyelination in the CNS. Neuron. 2015;85(6):1227-43. 
60. Agoston H, Khan S, James CG, Gillespie JR, Serra R, Stanton LA, et al. C-type natriuretic peptide regulates endochondral bone growth through p38 MAP kinase-dependent and -independent pathways. BMC Dev Biol. 2007;7:18.

61. Dauphinee SM, Eva MM, Yuki KE, Herman M, Vidal SM, Malo D. Characterization of two ENU-induced mutations affecting mouse skeletal morphology. G3 (Bethesda). 2013;3(10):1753-8.

62. Matsukawa N, Grzesik WJ, Takahashi N, Pandey KN, Pang S, Yamauchi M, et al. The natriuretic peptide clearance receptor locally modulates the physiological effects of the natriuretic peptide system. Proc Natl Acad Sci U S A. 1999;96(13):7403-8.

63. Moffatt P, Thomas G, Sellin K, Bessette MC, Lafreniere F, Akhouayri O, et al. Osteocrin is a specific ligand of the natriuretic Peptide clearance receptor that modulates bone growth. J Biol Chem. 2007;282(50):36454-62.

64. Gregson CL, Newell F, Leo PJ, Clark GR, Paternoster L, Marshall M, et al. Genome-wide association study of extreme high bone mass: Contribution of common genetic variation to extreme BMD phenotypes and potential novel BMD-associated genes. Bone. 2018;114:62-71.

65. Wang J, Tong KS, Wong LL, Liew OW, Raghuram D, Richards AM, et al. MicroRNA-143 modulates the expression of Natriuretic Peptide Receptor 3 in cardiac cells. Sci Rep. 2018;8(1):7055. 66. Ehrlich KC, Lacey M, Ehrlich M. Tissue-specific epigenetics of atherosclerosis-related ANGPT and ANGPTL genes. Epigenomics. 2019;11(2):169-86.

67. Tachmazidou I, Suveges D, Min JL, Ritchie GRS, Steinberg J, Walter K, et al. Whole-Genome Sequencing Coupled to Imputation Discovers Genetic Signals for Anthropometric Traits. Am J Hum Genet. 2017;100(6):865-84.

68. Zhang Y, Liu Y, Wu M, Wang H, Wu L, Xu B, et al. MicroRNA-664a-5p promotes osteogenic differentiation of human bone marrow-derived mesenchymal stem cells by directly downregulating HMGA2. Biochem Biophys Res Commun. 2020;521(1):9-14.

69. Zhang S, Mo Q, Wang X. Oncological role of HMGA2 (Review). Int J Oncol. 2019;55(4):775-88. 70. Yin X, Zhou C, Li J, Liu R, Shi B, Yuan Q, et al. Autophagy in bone homeostasis and the onset of osteoporosis. Bone Res. 2019;7:28.

71. Sgarra R, Pegoraro S, D'Angelo D, Ros G, Zanin R, Sgubin M, et al. High Mobility Group A (HMGA): Chromatin Nodes Controlled by a Knotty miRNA Network. Int J Mol Sci. 2020;21(3).

72. Zhang Y, Jin T, Shen H, Yan J, Guan M, Jin X. Identification of Long Non-Coding RNA Expression Profiles and Co-Expression Genes in Thyroid Carcinoma Based on The Cancer Genome Atlas (TCGA) Database. Med Sci Monit. 2019;25:9752-69.

73. Leszinski GS, Warncke K, Hoefele J, Wagner M. A case report and review of the literature indicate that HMGA2 should be added as a disease gene for Silver-Russell syndrome. Gene. 2018;663:110-4.

74. Stacchiotti S, Zuco V, Tortoreto M, Cominetti D, Frezza AM, Percio S, et al. Comparative Assessment of Antitumor Effects and Autophagy Induction as a Resistance Mechanism by Cytotoxics and EZH2 Inhibition in INI1-Negative Epithelioid Sarcoma Patient-Derived Xenograft. Cancers (Basel). 2019;11(7).

75. Yang F, Zhao L, Mei D, Jiang L, Geng C, Li Q, et al. HMGA2 plays an important role in Cr (VI)induced autophagy. Int J Cancer. 2017;141(5):986-97. 


\begin{tabular}{|c|c|c|c|c|c|c|c|c|}
\hline \multirow[b]{2}{*}{ Gene } & \multirow[b]{2}{*}{$\begin{array}{l}\text { Tier-1 SNP for } \\
\text { BMD association }\end{array}$} & \multirow{2}{*}{$\begin{array}{l}\text { Index/proxy } \\
\text { (I/P) or imputed } \\
\text { (Imp) SNP }\end{array}$} & \multirow{2}{*}{$\begin{array}{l}\text { Distance of } \\
\text { SNP to gene } \\
\text { TSS (kb) }\end{array}$} & \multirow[b]{2}{*}{$\begin{array}{c}\text { Alleles } \\
\text { (Ref/Alt) }\end{array}$} & \multirow[b]{2}{*}{$\begin{array}{l}\text { BMD-increasing } \\
\text { allele (Freq EUR) }\end{array}$} & \multirow[b]{2}{*}{$\begin{array}{l}\text { Chromatin state }^{c} \\
\text { at the SNP in ostb }\end{array}$} & \multicolumn{2}{|c|}{ Predicted TF binding ${ }^{d}$} \\
\hline & & & & & & & Ref allele & Alt allele \\
\hline \multirow[t]{7}{*}{ BICC1 } & rs112597538 & $\mathrm{I} / \mathrm{P}$ & -0.1 & $\mathrm{~T} / \mathrm{C}$ & Unknown (0.50) & Str prom & SREBP1/2 & None \\
\hline & rs1896245 & $\mathrm{I} / \mathrm{P} \& \mathrm{Imp}$ & 3.2 & $T / G$ & Alt $(0.50)$ & Str enh/prom & SATB1 & None \\
\hline & rs1896243 & $\mathrm{I} / \mathrm{P} \& \mathrm{Imp}$ & 3.5 & $\mathrm{C} / \mathrm{T}$ & Alt $(0.50)$ & Str enh & None & TCF3 \\
\hline & rs1658429 & I/P \& Imp & 57.6 & $\mathrm{~T} / \mathrm{C}$ & $\operatorname{Ref}(0.50)$ & Str enh & None & AP-1 \\
\hline & rs11006188 & Imp & 60.6 & $\mathrm{G} / \mathrm{C}$ & $\operatorname{Ref}(0.60)$ & Str enh & SMAD, GLIS3 & NR2F6 \\
\hline & rs1982173 & Imp & 61.2 & $\mathrm{G} / \mathrm{T}$ & $\operatorname{Ref}(0.60)$ & Str enh & RBPJ & None \\
\hline & rs4948523 & I/P \& Imp & 66.3 & $A / C$ & $\operatorname{Ref}(0.49)$ & Str enh & PRDX2 & None \\
\hline \multirow[t]{2}{*}{ NPR3 } & rs1173771 & $\mathrm{I} / \mathrm{P}$ & 104.3 & $A / G$ & Alt (0.58) & Strenh & SATB1 & None \\
\hline & rs7733331 & $\mathrm{I} / \mathrm{P}$ & 118.1 & $\mathrm{~T} / \mathrm{C}$ & Alt $(0.58)$ & Str enh & GTF2I & None \\
\hline LGR4 & rs10835153 & $\mathrm{I} / \mathrm{P} \& \mathrm{Imp}$ & -181 & $\mathrm{~A} / \mathrm{T}$ & $\operatorname{Ref}(0.41)$ & Str enh & None & BPTF \\
\hline \multirow[t]{3}{*}{ HMGA2 } & rs80019710 & Imp & -231.1 & $\mathrm{C} / \mathrm{T}$ & Alt (0.05) & Strenh & None & HMGA1 \\
\hline & rs17101510 & I/P \& Imp & -231 & $\mathrm{C} / \mathrm{T}$ & Alt (0.02) & Str enh & None & YY1 \\
\hline & rs12296417 & I/P \& Imp & -230.5 & $T / A$ & Alt (0.02) & Str enh & None & SATB1, ZBTB20 \\
\hline \multirow[t]{3}{*}{ DAAM2 } & rs2504105 & I/P \& Imp & 62.4 & $A / G$ & $\operatorname{Ref}(0.40)$ & Str enh/prom & SMAD & None \\
\hline & rs2504104 & I/P \& Imp & 62.4 & $A / G$ & $\operatorname{Ref}(0.40)$ & Str enh/prom & None & RUNX2/1, CBFB \\
\hline & rs2504103 & $\mathrm{I} / \mathrm{P}, \mathrm{Imp}$ & 62.5 & $C / G$ & $\operatorname{Ref}(0.40)$ & Str enh/prom & None & NFIC \\
\hline
\end{tabular}

${ }^{a} 16$ Tier-1 SNPs (prioritized candidates for regulatory osteoporosis-risk SNPs) and their 5 associated genes determined from 38 BMD GWAS. TSS, transcription start site; ostb, osteoblasts; TF, transcription factor; I/P, index or proxy SNP; Imp, imputed SNP; Ref, reference allele; Alt, alternate allele; Str enh, strong enhancer chromatin; Str prom, strong promoter chromatin

${ }^{\mathrm{b}}$ The BMD-increasing alleles and their frequency in the European population

'From Roadmap 18-State/Auxiliary Hidden Markov Model, States 1, 3, 8 or 9

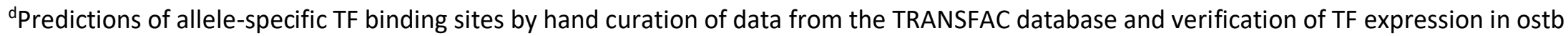

